\title{
EDITORIAL
}

\section{New ways in respiratory genetics}

\author{
M. Kabesch*, F. Kauffmann ${ }^{\#}$ and E. von Mutius*
}

D ver since the first reports on a genetic background for asthma, our knowledge about the role of genetic susceptibility in the development of respiratory diseases has increased steadily, as reviewed recently [1]. However, expectations of a fast victory in discovering the important genes for asthma and chronic obstructive pulmonary disease (COPD), respectively, and thereby find the true mechanisms underlying the development of these conditions, have faltered. At the same time, a sobering process has taken place allowing for a more realistic view on the status, the potential and the future of respiratory genetics. It cannot be disputed that significant progress has been achieved in this field. However, it is also true that with this increasing knowledge new scientific questions have arisen and that the transfer of genetic progress into clinical application is still awaited.

A highly stimulating European Respiratory Society meeting of leading groups in respiratory genetics and epidemiology from Europe and the USA, which took place in France in 2002 [2], eventually triggered a new European Respiratory Journal series "Respiratory genetics in the post-genome era", which aims to disseminate new concepts and ideas. Intentionally, this series does not provide another state-of-the-art review. Instead, leaders in the field were asked to communicate their personal views on some of the burning questions in respiratory genetics. A series of essays has emerged from this request in which selected authors present their personal interpretation of the available data on respiratory genomics and genetics. The authors also provide an educated guess as to how the future of genetic research in respiratory diseases may develop over the coming years.

In this issue, the series starts with a paper by LE SOUËF et al. [3], in which the authors argue that evolution may have more to do with asthma genetics than initially expected. They make a case that leaving tropical environments on the way "out of Africa" early in the development of mankind, may have contributed to the genetic susceptibility to develop asthma in modern societies. The large increase of asthma and allergy in developing countries is now a big concern for the future. In

*University Children's Hospital, Ludwig Maximilians University, Munich, Germany. "Inserm, U780 Epidemiology and Biostatistics and Université Paris Sud, Faculté de Médecine, Villejuif, France.

SUPPORT STATEMENT: The editors of the series are supported by the European Union Framework programme for research, contract No. F00D-CT-2004-506378, the Global Allergy and Asthma European Network $\left(\mathrm{GA}^{2} \mathrm{LEN}\right)$ project.

CORRESPONDENCE: M. Kabesch, University Children's Hospital, Ludwig Maximilians University Munich, Lindwurmstrasse 4, D-80337 Munich, Germany. Fax: 498951604764. E-mail: Michael.Kabesch@med.uni-muenchen.de the next issue, MARTINEZ [4] will address gene by environment interactions from yet another and unconventional perspective. Less evolutionary but no less intriguingly, MARTINEZ [4] will put forward the argument that environment is key to understanding genetics. MARTINEZ [4] reasons that it seems surprising that genetic associations have been identified at all so far if environmental influences have been disregarded. Considering genotype in isolation may lead to a dead end. In contrast, a network of genetic factors determining disease susceptibility may exist, which is present in an individual but will only lead to visible effects (and disease) when certain environmental triggers are also present. Such a model may explain why replication in genetic studies is so hard to achieve and underlines the need for multidisciplinary approaches to make sense of genetic data.

HOLGATE [5] revisits the genetics of structural cells and their remodelling as a key feature of asthma and other pulmonary diseases by taking ADAM33 as an example. While many genetic studies of asthma focus on molecules and genes that are involved predominantly in inflammation and immunomodulation, the positional cloning of ADAM33 opened a new window in respiratory research. The people who discovered the role of ADAM33 in asthma describe where the long and winding road has taken us so far, beginning with the positional cloning of the ADAM33 gene, and going backwards from its role in bronchial hyperresponsiveness and remodelling, to its possible role in modelling events during airways branching development.

SHAPIRO [6] takes a critical look at animal models and their use in respiratory genetics. Even though translation from mouse to human requires caution, a lot can be learned from transgenic and gene-targeted mice for respiratory diseases. Cell-specific transgenic mice models allow very specific pathways to be distinguished. Using examples from the field of COPD, SHAPIRO [6] illustrates how animal models can be used to dissect disease pathways in vivo and have already increased our knowledge about genetic influences in lung structure and development.

KOPPELMAN et al. [7] analyse the conditions under which genetic testing for asthma could become common practice in the future. In the absence of a gold standard in asthma diagnosis (especially in early childhood) a simple and inexpensive test to diagnose or even predict the development of asthma would be very much appreciated. Currently, genetic testing in complex diseases does not seem feasible, but disease prediction might become possible after thorough validation by combining information on personal genetic and environmental risk factors. Based on the experience with genetic testing in 
other fields of medicine, the authors explain what may be expected from genetic testing in asthma in the near future.

In a further part of the series, HALL and SAYERS [8] describe the progress and current status of pharmacogenetics in respiratory diseases. Whilst this potential application of genetics in respiratory diseases appears hopeful and its clinical application seems possible in the near future, the authors also point out the many difficulties that need to be overcome every step of the way, from identifying genetic variation in a gene involved in a drug pathway to its validation in clinical studies.

MOFFATT and COOKSON [9] take us to the edge of post-genome genetics as we know it now and risk a look into the future of what may and what may not be technically possible. They analyse the incredible progress that has been achieved in systematic genetics over recent years in programmes of worldwide cooperation, such as the human genome (HUGO) and the currently running Haplotype Map (HapMap) project, which will increase our knowledge on common human genetic variants dramatically. They are in favour of a systematic identification of genes involved in respiratory diseases. The next generation of studies could be based on whole-genome association and linkage studies in combination with disease phenotypes based on gene expression levels. MOFFATT and COOKSON [9] discuss the benefit of these developments and give an update of what kind of strategies, study designs and statistical issues may soon be expected in respiratory genetics.

Finally, CAMBON-THOMSEN et al. [10] address ethical issues that arise from the use of large-scale collections of biomaterials amassed in biobanks together with personal and clinical data of individuals under study. While the use of biobanks is a crucial component for the new generation of genetic studies now emerging on national and international levels, legal frameworks differ significantly between countries. The need for standardisation is evident and current pressing ethical issues such as protection of personality rights, sharing of data and the right of the public to adequate information are discussed in the last manusript of the series [10].
Overall, new directions in genetics need continuous refinement of phenotypic characterisation, with new approaches to characterising subjects biologically and environmentally over their life span. Collaborations should be developed within disciplines to generate sufficient power and to address the underlying complexity of chronic respiratory diseases, while at the same time, ethical questions arising from these large-scale studies need special consideration. We hope the readers enjoy this series of essays as much as the editors did. It is a "must read" for everyone interested in genetic medicine and the future of respiratory genetics.

\section{REFERENCES}

1 Silverman EK, Shapiro SD, Lomas DA, Weiss ST, eds. Respiratory Genetics. London, Edward Arnold, 2005; p. 527.

2 Kauffmann F, the Post Genome Respiratory Epidemiology group. Post-genome respiratory epidemiology: a multidisciplinary challenge. Eur Respir J 2004; 24: 471-480.

3 Le Souëf PN, Candelaria P, Goldblatt J. Evolution and respiratory genetics. Eur Respir J 2006; 28: 1258-1263.

4 Martinez FM. Genes, environments, development and asthma: a reappraisal. Eur Respir J 2007; (In press).

5 Holgate S. Aberrant epithelial mesenchymal communication in the origin and progression of asthma. Eur Respir J 2007; (In press).

6 Shapiro SD. Transgenic and gene targeted mice as models for COPD. Eur Respir J 2007; (In press).

7 Koppelman GH, te Meerman GJ, Postma DS. Genetic testing for asthma. Eur Respir J 2007; (In press).

8 Hall IP, Sayers I. Pharmacogenetics and asthma: false hope or new dawn? Eur Respir J 2007; (In press).

9 Moffatt MF, Cookson WOC. After the deluge: post-genome genetics of asthma and COPD. Eur Respir J 2007; (In press).

10 Cambon-Thomasen A, Rial-Sebbag E, Knoppers BA. Trends in ethical and legal framework for the use of human biobanks. Eur Respir J 2007; (In press). 